\title{
Interactive effects in transfer-appropriate processing for event-based prospective memory: The roles of effort, ongoing task, and PM cue properties
}

\author{
Drew H. Abney • Dawn M. McBride • Samantha N. Petrella
}

Published online: 7 May 2013

(C) Psychonomic Society, Inc. 2013

\begin{abstract}
Past studies (e.g., Marsh, Hicks, \& Cook Journal of Experimental Psychology: Learning, Memory, and Cognition 31:68-75, 2005; Meiser \& Schult European Journal of Cognitive Psychology 20:290-311, 2008) have shown that transfer-appropriate processing (TAP) effects in event-based prospective memory (PM) depend on the effort directed toward the ongoing task. In the present study, we addressed mixed findings from these studies and examined monitoring in TAP and transfer-inappropriate processing (TIP) conditions. In two experiments, a semantic or orthographic ongoing task was paired with a PM cue that either was matched in processing (TAP) or did not match in processing (TIP). Within each condition, effort was varied across trials. The results indicated that PM accuracy was higher in TAP than in TIP conditions, regardless of effort condition, supporting the findings reported by Meiser and Schult. Ex-Gaussian functions were fit to the mean reaction times (cf. Brewer Journal of Psychology $219: 117-124,2011)$ in order to examine monitoring across conditions. The analysis of distributional skew ( $\tau$ parameter) showed sensitivity to ongoing task instructions and properties of the PM cues. These results support Meiser and Schult's suggestion that TIP conditions require more attentional processing, and they also afford novel discussion on the interactive effects of ongoing task condition, PM cue properties, and manipulations of effort.
\end{abstract}

Keywords Prospective memory · Processing match . Distributional analysis

D. H. Abney • D. M. McBride · S. N. Petrella

Illinois State University, Normal, IL, USA

D. M. McBride $(\bowtie)$

Department of Psychology, Illinois State University, Campus Box 4620, Normal, IL 61790-4620, USA

e-mail: dmmcbri@ilstu.edu
Prospective memory (PM) involves remembering to perform an intended task at either the occurrence of a certain event or at a specific time, defined as event-based and time-based PM, respectively (Einstein \& McDaniel, 1990). The present study focused on event-based PM. Many laboratory investigations of event-based PM have focused on factors that aid or hinder executing delayed intentions (Ellis, 1996) and have typically examined PM performance by having participants perform an ongoing task while embedding an additional PM task. For example, participants may be asked to perform the task of determining if letter strings are words or nonwords in addition to the task of pressing a specific key upon detection of a specific word or a certain type of word.

Relying on the popular notion of transfer-appropriate processing (TAP; e.g., Morris, Bransford, \& Franks, 1977) that has been shown in numerous studies to affect retrospective memory (see Roediger, Weldon, \& Challis, 1989), several PM studies (e.g., Maylor, 1996, 1998) have suggested that degree of processing overlap between the ongoing task and the PM task influences successful PM cue detection. The degree of overlap is typically defined as the degree to which the ongoing task allows for automatic processing of the relevant features of the PM cues. For example, if the PM task is to respond to animal words, a lexical decision ongoing task would allow for one to notice that items identified as words are also animal words. In this way, the processing needed for the lexical decision task overlaps with processing needed to identify PM cues (see Marsh, Hicks, \& Cook, 2005). To test these ideas, Meier and Graf (2000) compared PM performance in conditions in which the ongoing tasks either matched the type of processing involved in the PM task or did not match the processing. Regardless of the type of processing involved (semantic or orthographic), PM performance was superior in conditions with a processing match. These results were also supported by findings reported by 
McGann, Ellis, and Milne (2003) and partially supported by findings reported by Marsh, Hicks, and Hancock (2000) and West and Craik (2001).

The way in which processing match between ongoing and PM tasks has been defined in past studies (e.g., Marsh et al., 2005; Maylor, 1996, 1998) can be viewed as a weak form of focal processing as defined by Einstein and McDaniel (2005). Focal processing occurs when the ongoing task focuses processing on the features of the target item that are relevant for the PM task. This is similar to the definition of a match in processing across the tasks described above, but requires that the feature of the PM cue that defines it as such be processed during the ongoing task. For example, with specific words as PM cues, a lexical decision ongoing task is a focal task because processing of the items for a word/nonword decision requires processing a particular property of the word. However, a PM task to respond to animal words in a lexical decision task is not a focal task because word/nonword decisions do not require processing of the category of the words.

If one considers focal tasks as a strong form of match in processing, then there is ample evidence that focal tasks show an advantage in PM task performance. Einstein et al. (2005) found higher PM performance for focal than nonfocal tasks in multiple experiments, and McBride and Abney (2012) found higher PM performance in their focal task than two comparison nonfocal tasks. These studies showing a PM advantage for focal over nonfocal tasks provide further evidence that a match in processing for ongoing and PM tasks aids PM task performance.

Marsh et al. (2005) provided additional support for a TAP view of PM performance, but they also investigated the interaction between TAP effects and effects of effort on the ongoing task. They reasoned that sufficient attentional resources (as are available under low effort conditions typically required in the ongoing task in many PM studies) are necessary to detect PM cues under TAP conditions due to reliance on the same type of processing across the ongoing and PM tasks in the matched processing conditions. When both tasks require similar processing, more attentional resources are consumed when completing the two tasks concurrently. To test this prediction, they examined effects of effort (i.e., low, medium, and high levels of speed and accuracy) toward the ongoing task. They found that TAP conditions resulted in higher PM performance than transfer-inappropriate (TIP) conditions only under low effort conditions when sufficient resources are available for both tasks. When more attention was devoted to the ongoing task in high-effort conditions, there was no advantage for tasks that overlapped in processing. From these results, Marsh et al. argued that a TAP advantage only occurs if there are sufficient attentional resources. When there is a decrement in attentional resources (e.g., increased effort to the ongoing task), PM cue detection decreases to levels seen in TIP conditions because the advantage given by the overlap in processing in the tasks can no longer be relied upon for PM task completion. In addition, a comparison of the ongoing task reaction times (RTs) between the two PM conditions (TAP and TIP) and a control condition in which participants completed the ongoing task without a PM task indicated slower ongoing task performance in the two PM conditions, with the TIP condition resulting in the slowest performance overall. These results suggest that more PM cost (Smith, 2003) occurred when processing type did not match for the ongoing and PM tasks, a finding consistent with more reliance on attentional resources overall in the TIP condition than in the TAP condition. However, no interaction between condition and effort was found, indicating that the interaction found between condition and effort for PM accuracy was not tied to an analogous PM cost in the ongoing task.

Meiser and Schult (2008) provided a similar test of the interaction between TAP conditions and effort. However, they suggested that Marsh et al.'s (2005) results were likely due to the nature of their effort manipulation. They argued that manipulating effort by varying both speed and accuracy might have confounded processing such that increased speed "impairs strategic processing" and increased accuracy supports "cue detection by careful processing of the target stimuli" (p. 294). Thus, in their study, Meiser and Schult manipulated available attentional resources by varying both the amount and type of effort toward the ongoing task. Participants were instructed to respond to ongoing task trials with low, medium, or high effort, with effort equated to speed or accuracy in different groups of participants. Meiser and Schult's predictions were supported with PM performance showing a TAP advantage with speed instructions but similar performance in TAP and TIP conditions with accuracy instructions. In other words, the TAP effect depended on what aspect of ongoing task performance, in terms of speed or accuracy, was focused on in the effort manipulation. Their interpretation of the results was that high speed-effort responses in the ongoing task depleted attentional resources such that the relatively high PM performance seen in the TAP condition reflected heavier reliance on more automatic forms of processing. Thus, in contrast to Marsh et al.'s claim that attentional resources are required for a TAP advantage, these results support Meiser and Schult's suggestion that TAP advantages in PM tasks do not always rely on attentional resources (see McGann, Ellis, \& Milne, 2002, for a similar argument). A control condition without the PM task was not included in this study to examine PM cost as in Marsh et al.'s study, but correlational analyses showed longer RTs connected with higher PM accuracy only in the TIP condition with accuracy instructions. No such correlation was seen in the TIP condition with speed instructions (or in either of the TAP conditions in which PM accuracy was high) indicating that the lower PM accuracy in this condition was a result of having fewer attentional resources available that are needed for the 
task. Thus, Meiser and Schult's results stand in direct conflict with Marsh et al.'s claim that TAP effects rely on attentional resources.

The present study was designed to further investigate Marsh et al.'s (2005) suggestion regarding reliance on attentional resources for PM performance in TAP conditions. In light of the discrepancy in TAP/effort interactive effects in event-based PM tasks across Marsh et al.'s and Meiser and Schult's (2008) studies and the importance of the use of attentional resources in current views of PM performance (e.g., the preparatory attention view (Smith, 2003) and the multiprocess view (McDaniel \& Einstein, 2000)), the present study was designed to further investigate this interaction effect and measure PM cost in these conditions using withinsubjects (Exp. 1) and between-subjects (Exp. 2) designs to manipulate ongoing task and PM cue properties. In two experiments, we sought to replicate Meiser and Schult's results showing a TAP advantage under both low- and higheffort conditions in which effort was equated with speed in the ongoing task (henceforth, low-speed-effort, medium-speedeffort, and high-speed-effort). Such results might provide support that TAP effects do not rely on attentional resources when little to no PM cost is seen in these conditions, and also, that conditions with TIP effects (and potentially other interaction effects) might rely on more attentional resources such as strategic monitoring. For Experiment 1, the measurement of PM cost to the ongoing task with within-subjects baseline trials allowed for a comparison of PM cost in TAP and TIP conditions for each participant. If high-PM performance is found in the TAP conditions with less accompanying PM cost to the ongoing task than in TIP conditions, this will further support Meiser and Schult's suggestion that TAP effects do not always rely on attentional resources. In Experiment 2, a separate control group that did not complete the PM task was included to evaluate speed cost in the second block of trials that was due to the PM task.

For Experiment 1, match in processing between ongoing and PM tasks was manipulated in an experiment involving three blocks of ongoing task trials - baseline block, TAP PM block, and TIP PM block-in which the order of the PM blocks was counterbalanced. Different PM tasks were used to create the TAP and TIP conditions to allow for within-subjects comparison of speed for the same ongoing task across baseline, TAP, and TIP conditions, as examination of attentional resources required for the task was a primary purpose of the present study. Thus, the completely within-subjects design allowed for within-subjects comparisons of ongoing task speed for the same ongoing task. The counterbalancing of PM task condition spread practice effects equally across these two orders. The ongoing task involved lexical decisions task, as this was the ongoing task used by both Marsh et al. (2005) and Meiser and Schult (2008). The TAP PM task involved responding to animal words (i.e., semantic task). The TIP PM task involved responding to palindromes (i.e., orthographic task).

In Experiment 2, we attempted to generalize the findings of Experiment 1 to different ongoing tasks, one semantic task (living/nonliving judgments) and one orthographic task (consecutive-letter judgments). Each ongoing task was paired with a semantic (respond to animal or bird items) and an orthographic (respond to palindromes) PM task to allow for both semantic and orthographic TAP conditions.

PM task cost was tested with standard analyses of RTs and with a distributional analysis fitting ex-Gaussian functions to RT data, as was suggested by Brewer (2011). The ex-Gaussian function fits estimated $\mu, \sigma$, and $\tau$ parameters for each participant and then parameters were separately analyzed to determine effects of TAP condition and effort (with Ongoing Task Condition as an additional factor in Exp. 2) on each aspect of the RT distribution. Although standard analyses of mean RTs have been common utility for PM researchers, fitting statistical distributions to empirical RT data affords the opportunity to decompose the empirical RT distribution into various (and more) components (Brewer, 2011; see Balota \& Yap, 2011, for another recent example of this argument). The ex-Gaussian function was chosen because it has been shown to provide good fits to RT distributions (e.g., Brewer, 2011; Heathcote, Popiel, \& Mewhort, 1991; McBride \& Abney, 2012; Tse, Balota, Yap, Duchek, \& McCabe, 2010) due to a commonly found skew in the slow tail of the distribution (Luce, 1986).

Ex-Gaussian function fits were performed using the Quantile Maximum Probability Estimation (QMPE) program (Cousineau, Brown, \& Heathcote, 2004; Heathcote, Brown, \& Cousineau, 2004) that estimated three parameters for each participant's block by effort RT data: $\mu, \sigma$, and the exponential parameter $\tau$. The $\mu$ and $\sigma$ parameters provide estimates of the mean and standard deviation of the distribution, whereas, the $\tau$ parameter provides an estimate of the skew of the distribution. Importantly, the $\mu$ and $\sigma$ parameters of the ex-Gaussian distribution are components of the sample mean (i.e., mean computed from the Gaussian distribution). For example, if the $\mu$ is $700 \mathrm{~ms}$ and the $\sigma$ is $300 \mathrm{~ms}$, the sample mean would be approximately 1,000 ms. Thus, a direct comparison between the sample mean and the estimated $\mu$ is not advocated or necessarily accurate.

Examining multiple parameters that describe ongoing task speed allows us to assess possible differential effects of manipulations of processing overlap and attention allocation on different aspects of ongoing task speed. For example, in a PM task, Brewer (2011) found no difference in $\mu$ across blocks (baseline and PM blocks), but found that $\tau$ was larger for PM task blocks, indicating that although there was no mean shift in the distribution due to the addition of a PM task (in $\mu$, not sample mean), more skewing was found in the slow tail, indicating 
higher frequency of slower responses during the PM task under conditions that produced no difference in $\mu$ estimates. Thus, the distributional analysis can provide additional information regarding the type of PM cost involved in the conditions above and beyond the information provided in traditional mean RT analyses.

One common question about the ex-Gaussian parameter estimates regards the affordance of insight toward the cognitive processes at play during experimental tasks. Although tentative in the literature, distinctions have been made that assert that the Gaussian components of the distribution (i.e., $\mu$ and $\sigma$ ) reflect automatic-like processes and the exponential component (i.e., $\tau$ ) reflects controlled-like, analytic processes (Hohle, 1965; for an extended discussion, see also Balota \& Spieler, 1999). We share the cautious demeanor regarding mapping distribution parameters to cognitive processes (Hohle, 1965; McGill \& Gibbon, 1965; Luce, 1986; Matzke \& Wagenmakers, 2009); however, for the present study's goals and methodology, we find it warranted to provide tentative hypotheses regarding the potential outcomes of the distributional analysis. As Brewer (2011) found, we also expect that the skew of the distribution (i.e., $\tau$ ) will be sensitive to PM costs. Furthermore, the experimental design in the present study affords investigating the sensitivity of the $\tau$ parameter beyond what has already been found (e.g., a traditional PM cost, Brewer, 2011). Particularly, our design provides the potential to observe differential effects of the distributional parameters as a function of not just PM condition properties, but also of TAP condition properties and effort manipulations - such an investigation is without precedence in the PM literature. Such observations of the differential sensitivity of the $\tau$ parameter can afford tentative discussion regarding the controlled (i.e., analytic) processes (Hohle, 1965; Luce, 1986) that are utilized in difficult task conditions for successful PM performance.

\section{Experiment 1}

Method

Participants and design A group of 47 undergraduates (42 female, five male) from Illinois State University participated and received course credit. All of the participants were fluent English speakers. Seven were removed from the study for failure to follow task instructions $(n=5)$ or not remembering instructions at the end of experiment $(n=2)$; thus, the analyses focused on the remaining 40 participants. The experimental design comprised of three within-subjects prospective memory conditions (baseline block, semantic PM block, orthographic PM block) with semantic and orthographic block order counterbalanced across participants. Accuracy for both ongoing task trials and PM task trials and reaction time (RT) to complete ongoing task trials were recorded for each participant.

Materials and procedure Stimuli consisted of letter strings that appeared in the center of a black screen in white, Times New Roman font. All letter strings were presented in uppercase lettering. We obtained 156 words chosen from exemplars in the Kučera and Francis (1967) normative compendium, 162 nonwords from exemplars in the ARC Nonword Database (Rastle, Harrington, \& Coltheart, 2002), and six palindromes from exemplars in the Chism (1992) palindrome compendium. All words and nonwords were matched for frequency and letter range (four to six). PM cue stimulus properties (i.e., animals and palindromes) were not used in the stimulus set in the lexical decision task. For the baseline block, 54 words and 54 nonwords were presented in random order. For both PM blocks, 48 words and 54 nonwords were presented in random order, and six PM cues (varied by PM task block) were presented in a fixed order (Trials 26, 44, 62, 80, 98, and 104).

Participants were instructed that they were taking part in an experiment with multiple phases and instructions for each phase would be given before beginning that phase. In the first phase, all participants performed the baseline block of the ongoing task. In the baseline block, participants were instructed to perform a lexical decision task in which they had to decide if a letter string was a word or a nonword. Depending on counterbalancing condition, participants pressed " 1 " for word and "0" for nonword or vice versa.

Effort was manipulated by prompting participants with a label (LOW EFFORT, MEDIUM EFFORT, or HIGH EFFORT) before each triad of trials (cf. Meiser \& Schult, 2008). For lowspeed-effort trials, participants were instructed that they should respond to each letter string at a slow and relaxed pace. In contrast, for high-speed-effort trials, participants were instructed that they should respond to each letter string at a fast pace. For medium-speed-effort trials, participants were instructed to respond to each letter string with a speed that was in between low-speed- and high-speed-effort trials. Three PM cues were presented on low-speed-effort triads and three cues were presented in high-speed-effort triads. All cues were presented in the middle trial of each triad. The effort manipulation was blocked in a sinusoidal fashion (cf. Marsh et al., 2005) such that triplets of trials were sequenced as low-speed-effort, medium-speed-effort, high-speed-effort, medium-speed-effort, low-speed-effort, and so on, for 36 triads. After baseline block and effort manipulation instructions, all participants proceeded with a practice session in which three lexical decision trials at each speed-effort level were presented in order for participants to become comfortable with each speed-effort instruction. Each triad began with a fixation point for $250 \mathrm{~ms}$, a speed-effort prompt for $1,500 \mathrm{~ms}$, another fixation point for $250 \mathrm{~ms}$, and then three separate letter strings. Each letter 
string remained on the screen until a word or nonword response was endorsed, which was followed by an intertrial interval of $250 \mathrm{~ms}$ in which a fixation point was presented on the screen.

For the semantic PM block, in addition to the ongoing task and speed-effort manipulation instructions, participants were instructed to press the " $z$ " key instead of the " 1 " or " 0 " key if they encountered an animal word. The semantic cues included HORSE, MOUSE, BEAVER, LION, MONKEY, and BEAR. For the orthographic PM block, participants were given identical instructions except for the type of cues they were to respond to: They were instructed to press the " $\mathrm{z}$ " key instead of the " 1 " or " 0 " key if they encountered palindromes. Participants were instructed that palindromes were words that could be spelled the same way both forward and backward. The orthographic cues included TENET, ROTOR, LEVEL, KAYAK, SOLOS, and CIVIC. After instructions were provided and any questions were answered, participants engaged in solving Sudoku puzzles for $2 \mathrm{~min}$ as a distractor activity before beginning the ongoing task with the given PM task without any further discussion of the additional prospective memory task.

Results and discussion

PM accuracy PM cue detection was defined as the proportion of the three cues detected in each speed-effort condition for each PM task block (i.e., TAP and TIP PM blocks, independently). As is typical in PM studies, PM cue responses were considered correct if participants pressed the " $\mathrm{z}$ " key on the trial on which a PM cue was presented or the trial immediately following the cue (see Table 1 for the mean PM accuracy by conditions). False alarms to PM

Table 1 Mean reaction times (RTs, in milliseconds), PM cue detection, ongoing task accuracy, and standard errors (in parentheses) by PM task condition and speed-effort for Experiment 1

Mean RT PM Cue Detection Ongoing Task Accuracy

\begin{tabular}{lrll}
\hline $\begin{array}{l}\text { Baseline } \\
\text { Low }\end{array}$ & $1,020(24)-$ & $.93(.02)$ \\
Medium & $940(17)-$ & $.90(.02)$ \\
High & $830(23)-$ & $.85(.02)$ \\
TAP & & & \\
Low & $986(38)$ & $.80(.04)$ & $.94(.03)$ \\
Medium & $976(22)$ & - & $.93(.02)$ \\
High & $927(23)$ & $.75(.04)$ & $.91(.02)$ \\
TIP & & & $.92(.03)$ \\
Low & $1,200(41)$ & $.63(.05)$ & $.91(.03)$ \\
Medium & $1,112(30)$ & - & $.90(.03)$ \\
High & $1,065(32)$ & $.55(.04)$ & \\
\hline
\end{tabular}

cues were rare and did not affect the results. A 2 (PM task type: TAP vs. TIP) $\times 2$ (speed-effort: low vs. high) repeated measures analysis of variance (ANOVA) performed on proportion of PM cue detection showed a significant difference between PM tasks, $F(1,39)=23.48, p<.001, \eta_{\mathrm{p}}{ }^{2}=.376$. The TAP PM task $(M=.78, S E=.03)$ resulted in higher accuracy than the TIP PM task $(M=.59, S E=.03)$. This result is consistent with the TAP view (and some past studies supporting this view). This view suggests an advantage in PM accuracy when there is a processing match between the ongoing and PM tasks. As was reported by Meiser and Schult (2008), there was no interaction between PM task and speedeffort, $F(1,39)=.27, p=.61, \eta_{\mathrm{p}}^{2}=.007$. In addition, the main effect of speed-effort was not significant, $F(1,39)=2.75$, $p=.11, \eta_{\mathrm{p}}{ }^{2}=.066$.

Ongoing task accuracy The mean accuracy proportions on the ongoing task are presented in Table 1. All participants had ongoing task accuracy above an initial $80 \%$ criterion. In accordance with past studies (e.g., Marsh et al., 2005) only the low- and high-speed-effort conditions were included in the analyses to examine the effects of the largest speed difference, but means for all three speed-effort conditions are shown in Table 1. Thus, a 3 (PM task type: baseline vs. TAP vs. TIP) $\times 2$ (speed-effort: low vs. high) repeated measures ANOVA was performed on proportion of correct responses to ongoing task trials, and showed a main effect of speed-effort, $F(1,39)=10.86, p=.002, \eta_{\mathrm{p}}{ }^{2}=.218$, with higher accuracy in low-speed-effort trials $(M=.93, S E=.03)$ than in highspeed-effort trials $(M=.90, S E=.02)$. This result was expected due to the faster speed with which participants were asked to complete high-speed-effort ongoing task trials. However, ongoing task accuracy was high in both speed-effort conditions. Neither the main effect of PM task nor the interaction was significant for ongoing task accuracy, $p \mathrm{~s}>.30$.

Ongoing task speed Ongoing task speed was analyzed to examine conditions that produced task interference in the ongoing task. Trials on which an incorrect response was endorsed or when a PM cue was presented were excluded from RT analyses. Trials on which RTs were 2.5 standard deviations from the mean for each participant were trimmed, resulting in a deletion of $1.06 \%$ trials across all participants.

A 3 (block: control vs. TAP vs. TIP) $\times 2$ (speed-effort: low vs. high) repeated measures ANOVA performed on the RTs indicated a main effect of block, $F(2,78)=34.56$, $p<.001, \eta_{\mathrm{p}}{ }^{2}=.47$. Overall, trials in the control (i.e., Block 1, $M=925 \mathrm{~ms}, S E=19 \mathrm{~ms})$ and TAP $(M=956 \mathrm{~ms}, S E=26 \mathrm{~ms})$ blocks had faster RTs than trials in the TIP block $(M=1,132 \mathrm{~ms}, S E=33 \mathrm{~ms})$. A main effect of speed-effort, $F(1,39)=24.34, p<.001, \eta_{\mathrm{p}}{ }^{2}=.384$, showed that, overall, low-speed-effort trials $(M=1,068 \mathrm{~ms}, S E=29 \mathrm{~ms})$ 
were slower than high-speed-effort trials $(M=940 \mathrm{~ms}$, $S E=21 \mathrm{~ms}$ ). The main effects were qualified by a Block $\times$ Speed-Effort interaction, $F(2,78)=8.09, p=.001$, $\eta_{\mathrm{p}}{ }^{2}=.172$. Simple effects tests showed that, for low-speedeffort, trials in the control block $(M=1020 \mathrm{~ms}, S E=24 \mathrm{~ms})$ were faster than trials in the TIP block $(M=1,200 \mathrm{~ms}$, $S E=41 \mathrm{~ms}), p<.001$. Trials in the control and TAP ( $M=986 \mathrm{~ms}, S E=24 \mathrm{~ms})$ blocks for low-speed-effort were not different from each other, $p=.357$. For high-speedeffort trials, all blocks differed significantly from each other (all $p \mathrm{~s}<.002$ ), with trials in the control block $(M=830 \mathrm{~ms}, S E=23 \mathrm{~ms})$ being faster than those in the TAP block $(M=927 \mathrm{~ms}, S E=23 \mathrm{~ms})$, which were faster than those in the TIP block ( $M=1,065 \mathrm{~ms}, S E=32 \mathrm{~ms})$. The mean RTs by blocks are given in Table 1 .

$R T$ distribution analyses We submitted participant-level RTs to the QMPE program after the data were trimmed using the same procedure discussed above in the Ongoing Task Performance subsection. Mean parameter estimates are provided in Table 2. Parameter estimates $(\sigma, \mu$, and $\tau)$ were separately analyzed in 3 (PM task type: baseline vs. TAP vs. TIP) $\times 2$ (speed-effort: low vs. high) repeated-measures ANOVAs. The model fits converged for data sets for 29 of the 40 participants $(72.5 \%)$. Thus, we had a complete set (i.e., $\sigma, \mu$, and $\tau$ ) of parameter estimates for 29 participants. Upon further examination, PM accuracy for the restricted sample was consistent with initial findings in which TAP conditions had higher cue detection than TIP conditions, $F(1,27)=10.35, p=.003, \eta_{\mathrm{p}}{ }^{2}=.277$. The only difference between the original and restricted samples was that there was a marginal effect of speed-effort in the restricted sample, $F(1,27)=3.44, p=.075, \eta_{\mathrm{p}}{ }^{2}=.113$. No other effects or interactions were significant, all $F_{\mathrm{S}}<1$.

Results for the parameter estimate of standard deviation $(\sigma)$ showed no significant main effect of PM task type, $F(2,56)=$ $1.13, p=.331, \eta_{\mathrm{p}}{ }^{2}=.039$, or speed-effort, $F(1,28)=.01$, $p=.93, \eta_{\mathrm{p}}^{2}<.001$, and no significant interaction, $F(2,56)=$ $2.59, p=.084, \eta_{\mathrm{p}}{ }^{2}=.085$.

Table 2 Mean $\mu, \sigma$, and $\tau$ parameter estimates (in milliseconds), with standard errors (in parentheses), by PM task and speed-effort conditions for Experiment 1

\begin{tabular}{llrl}
\hline & \multicolumn{2}{l}{ Mean Parameter Estimates } \\
\cline { 2 - 4 } & $\mu$ & \multicolumn{1}{l}{$\tau$} \\
\hline Baseline/Low & $870(22)$ & $153(19)$ & $107(23)$ \\
Baseline/High & $714(20)$ & $99(20)$ & $119(16)$ \\
TAP/Low & $856(30)$ & $85(23)$ & $161(23)$ \\
TAP/High & $821(22)$ & $94(19)$ & $111(21)$ \\
TIP/Low & $868(40)$ & $88(26)$ & $287(38)$ \\
TIP/High & $796(27)$ & $138(30)$ & $233(31)$ \\
\hline
\end{tabular}

Results for the $\mu$ parameters differed from those of the mean RT analyses presented above. The main effect of PM task type, $F(2,56)=2.65, p=.080, \eta_{\mathrm{p}}^{2}=.086$, was not significant. However, the main effect of speed-effort, $F(1,28)=10.03$, $p=.004, \eta_{\mathrm{p}}{ }^{2}=.264$, (low-speed-effort trials: $M=865$, $S E=25$, high-speed-effort trials: $M=777, S E=17)$ and PM Task Type $\times$ Speed-Effort interaction, $F(2,56)=4.62$, $p=.014, \eta_{\mathrm{p}}{ }^{2}=.142$, were significant. Simple effects tests for the interaction showed no differences across PM task conditions for low-speed-effort trials, $p \mathrm{~s}>.65$, but faster baseline performance compared with both PM tasks for high-speedeffort trials, $p \mathrm{~s} \leq .008$. No difference was seen across the PM tasks with high-speed-effort, $p=.44$. Thus, the mean RTs in the distributions showed a PM task cost for both PM tasks in high-speed-effort trials, but no cost for either PM task with low-speed-effort.

The results for the $\tau$ parameter indicated a main effect of PM task type, $F(2,56)=19.09, p<.001, \eta_{\mathrm{p}}{ }^{2}=.405$, indicating differences in RT distribution skew across PM task conditions. Pairwise Comparisons showed that the TIP PM task type had higher frequencies of slow RTs (greater positive skew) than did either the baseline or the TAP PM condition, $p \mathrm{~s}<.001$. The main effect of speedeffort, $F(1,28)=2.72, p=.11, \eta_{\mathrm{p}}{ }^{2}=.089$, and the PM Task Type $\times$ Speed-Effort interaction, $F(2,56)=1.11$, $p=.34, \eta_{\mathrm{p}}{ }^{2}=.038$, were not significant. These results indicate a higher skew in the RT distributions only when there is a mismatch in processing (TIP condition), regardless of effort.

Importantly, we would be remiss to not point out a few differences in the results of the standard RT analyses and the distributional analyses. First, the nature of the QMPE (Cousineau et al., 2004) program is sensitive to the frequency of values used for each estimated partition (e.g., low-speedeffort response times from the TAP block). Although, for most trials, we stayed within the suggested range of frequency values ( $>40$ values), the efficacy of the parameter estimations drops when the frequency of values drops. Furthermore with many distributional analyses, including the QMPE, parameter estimation does not occur (i.e., converge) with low frequencies of input values. For Experiment 1, convergence issues for some trials were likely due to the RT trimming procedure discussed above. Second, it is important to point out again that the $\mu$ and the sample mean are separate parameters. Because the $\mu$ parameter is different (and a component) of the sample mean, the differences between the RT results in the sample mean and the results from the $\mu$ parameter should not be taken as an inconsistency but rather a difference in parameter or dependent measure.

\section{Experiment 2}

Experiment 2 was conducted in an attempt to generalize the results of Experiment 1 and also to investigate the effects of 
ongoing task type on PM performance. Participants were randomly assigned to one of six conditions that varied by ongoing task type and PM task type. The Ongoing Task Type factor consisted of either a semantic ongoing task or an orthographic ongoing task. Considering the nature of the two tasks, it is possible that PM performance and attentional processing are modulated by ongoing task type. The PM Task Type factor consisted of control, TAP, and TIP conditions, with specific properties of the TAP and TIP conditions varying as a function of the particular ongoing task type. The speed-effort manipulation was still implemented as a within-subjects factor. A finding of ongoing task type modulating the amount of $\mathrm{PM}$ performance, independent of the TAP effect, would provide further insight into the properties of successful PM performance.

\section{Method}

Participants and design Two hundred and two undergraduates from Illinois State University participated and received course credit for Experiment 2. None of the participants from Experiment 1 were included in this sample. All participants were fluent English speakers. Twenty participants were deleted from the study for: failure to follow task instructions $(n=8)$, not remembering instructions at the end of experiment $(n=10)$, or having poor ongoing task accuracy (less than $80 \%$ accuracy, $n=2$ ). Thus, the analyses focused on the remaining 182 participants. The experimental design comprised of six between-subjects prospective memory and ongoing task conditions (e.g., control, TAP, and TIP PM tasks for living/nonliving and consecutive-letter judgment tasks). For each condition, we created two conditions to reflect a counterbalancing of decision keypresses (i.e., for each condition, in one group, "yes" was endorsed with a "1" keypress, and in the other group, "yes" was endorsed with a "0" keypress). Accuracy for both ongoing task trials and PM-task trials, as well as RTs to complete the ongoing task trials, were recorded for each participant.

Materials and procedure Stimulus presentation and development were identical to Experiment 1. Participants were presented with two blocks of trials: (1) a baseline block and (2) a PM block. The main factors that were manipulated were Ongoing Task (semantic and orthographic) and PM-Task Condition (control, TAP, TIP). All participants were given ongoing task instructions to either (1) identify words with consecutive letters (i.e., orthographic ongoing task) or (2) identify words that were living/nonliving (i.e., semantic ongoing task).

For the consecutive-letters ongoing task, the participants in all conditions were presented with the same baseline block of trials. For the baseline block, 54 words with two consecutive letters (e.g., FUNNY, SOCCER) and 54 words without two consecutive letters (e.g., BASIN, SHADOw) were presented in random order. Participants assigned to the control condition were given a second block of trials with identical stimulus organization as the baseline block but with new words. Participants in the TAP-orthographic condition were given a second block of trials with 54 words with two consecutive letters, 48 words without two consecutive letters, and six palindrome words (TAP PM cues). Palindrome PM cues were identical to those used in Experiment 1. Participants in the TIP-semantic condition were given a second block of trials with 54 words with two consecutive letters, 48 words without two consecutive letters, and six animal words (TIP PM cues). Semantic PM cues were identical to those in Experiment 1.

For the living/nonliving ongoing task, participants in each condition were again presented with the same baseline block of trials. For the baseline block, 54 words were presented that were living objects (i.e., BANKER, DOG) and 54 words were presented that were nonliving objects (i.e., PENCIL, TABLE). Participants assigned to the Control condition were given a second block of trials with identical stimulus organization as the baseline block but with new words. Participants in the TAP-semantic condition were given a second block of trials with 54 living objects, 48 nonliving objects, and six types of birds (EAGLE, HAWK, PIGEON, BLUEJAY, FALCON, CARDINAL; TAP PM cues). Participants in the TIP-orthographic condition were given a second block of trials with 54 words that were living objects, 48 words that were nonliving and six palindrome words (TIP PM cues). Palindrome PM cues were identical to those used in Experiment 1 and those used in the consecutive-letters task described above. Unless discussed otherwise above, the speed-effort manipulation and PM task instructions were identical to Experiment 1.

\section{Results and discussion}

PM accuracy The definition of PM cue detection was identical to Experiment 1. Consistent with Experiment 1, false alarms to PM cues were rare and did not affect the results reported here. A 2 (PM task condition: TAP vs. TIP $) \times 2$ (PM task type: semantic vs. orthographic) $\times 2$ (speed-effort: low vs. high) mixed-measures ANOVA was conducted to test the hypothesis that TAP conditions provide an advantage to the PM task, regardless of type of PM task. The ANOVA showed a significant difference between PM task type, $F(1,115)=13.07, p<.001, \eta_{\mathrm{p}}{ }^{2}=.10,{ }^{1}$ indicating that the semantic PM tasks $(M=.73, S E=.03)$ resulted in higher accuracy overall than the orthographic PM task $(M=.53$, $S E=.03)$. In addition, the main effect of PM task condition

\footnotetext{
${ }^{1}$ Due to an experimenter error, the PM accuracy and ongoing task accuracy data were lost for one participant. Thus, one participant is not included in these analyses.
} 
was also significant, $F(1,115)=10.12, p=.002, \eta_{\mathrm{p}}{ }^{2}=.08$. The TAP PM task $(M=.71, S E=.03)$ resulted in higher accuracy than the TIP PM task $(M=.56, S E=.04)$. This result is consistent with the TAP view and with the results from Experiment 1. However, there was no significant interaction between PM task type and PM task condition, $F(1,115)=2.13, p=.148, \eta_{\mathrm{p}}{ }^{2}=.02$, and there were no effects of speed. Thus, these results indicate a TAP advantage in PM task performance for both PM tasks. This advantage was present for both speed-effort conditions, supporting the results of the present Experiment 1 and results reported by Meiser and Schult (2008).

To investigate the differential effects of the ongoing task type on PM performance, we conducted a 2 (ongoing task type: consecutive letters vs. living/nonliving $) \times 2$ (PM cue type: semantic vs. palindrome) $\times 2$ (speed-effort: low vs. high) mixed-measures ANOVA on the proportion of PM cue detection. A main effect of PM cue type, $F(1,115)=13.07$, $p<.001, \eta_{\mathrm{p}}{ }^{2}=.10$, indicated higher PM performance for semantic cues $(M=.72, S E=.05)$ than for palindrome cues $(M=.53, S E=.05)$. This effect was qualified by an Ongoing Task Type $\times$ PM Cue Type interaction, $F(1,115)=10.12$, $p=.002, \eta_{\mathrm{p}}{ }^{2}=.08$. Simple effects tests showed that, for palindrome PM cues, PM performance during the consecutiveletters ongoing task $(M=.65, S E=.05)$ was higher relative to the living/nonliving ongoing task $(M=.42, S E=.05), p=.001$. For semantic PM cues, there were no differences in PM performance for the consecutive-letters ongoing task $(M=.67$, $S E=.05)$ relative to the living/nonliving ongoing task $(M=.76$, $S E=.05), p=.231$. No other effects were significant, $p \mathrm{~s}>.1$.

Ongoing task accuracy The mean accuracy proportions on the ongoing task are presented in Table 3. Overall, ongoing task accuracy was high. For simplicity in interpretation, accuracy for each ongoing task was analyzed separately. A 2 (speed-effort: low vs. high) $\times 3$ (PM task condition: control vs. orthographic vs. semantic) $\times 2$ (block: 1 vs. 2 ) mixed-measures ANOVA was performed on the proportions of correct responses for each ongoing task. For the consecutive-letters task, a main effect of speed-effort was found, $F(1,87)=5.75, p=.019, \eta_{\mathrm{p}}^{2}=.06$, with higher accuracy for the low-speed-effort condition $(M=.96, S E=$ $.004)$ than the high-speed-effort condition, $(M=.94, S E=$ $.007)$, as was seen in Experiment 1. This effect was qualified by a Speed-Effort $\times$ PM Task Condition interaction, $F(2$, $87)=7.25, p=.001, \eta_{\mathrm{p}}{ }^{2}=.14$. We also found a three-way interaction between the factors, $F(2,87)=4.57$, $p=.013, \eta_{\mathrm{p}}{ }^{2}=.10$. No other effects were significant in this analysis, all $p \mathrm{~s}>.18$. For the living/nonliving task, however, the main effect of speed, $F(1,88)=5.31, p=.024, \eta_{\mathrm{p}}{ }^{2}=.06$, showed the opposite result: Accuracy was higher for the high-speed-effort condition $(M=.93, S E=.004)$ than for the low-speed-effort condition $(M=.92, S E=.005)$. In addition, a block main effect, $F(1,88)=39.90, p<.001, \eta_{\mathrm{p}}{ }^{2}=.31$, indicated higher accuracy in Block $2(M=.94, S E=.004)$ than in Block $1(M=.91, S E=.004)$. Finally, a marginally significant interaction between block and PM task condition was seen, $F(2,88)=2.91, p=.06, \eta_{\mathrm{p}}{ }^{2}=.06$. No other effects were significant in this analysis, all $p \mathrm{~s}>.23$.

Ongoing task speed The protocol for analyzing ongoing task speed and the computation of difference scores in Experiment 2 were identical to that in Experiment 1. Because the two different ongoing tasks may include different speed ranges, we conducted separate analyses for each ongoing task type.

For the consecutive-letters ongoing task (in which the palindrome PM cue was considered TAP), a 3 (PM task condition: control vs. TAP vs. TIP) $\times 2$ (block: 1 vs. 2$) \times 2$ (speed-effort: low vs. high) mixed-measures ANOVA with PM Task Condition as a between-subjects factor was performed on the RTs. A main effect of PM task condition, $F(2,88)=9.74, p<.001, \eta_{\mathrm{p}}{ }^{2}=.181$, showed that trials in the control block $(M=936 \mathrm{~ms}, S E=31 \mathrm{~ms})$ were faster than trials in both the TAP $(M=1,117 \mathrm{~ms}$, $S E=30 \mathrm{~ms})$ and TIP $(M=1,077 \mathrm{~ms}, S E=31 \mathrm{~ms})$ blocks, $p<.001$. A main effect of block, $F(1,88)=$ 116.10, $\left.p<.001, \eta_{\mathrm{p}}{ }^{2}=.569\right)$, showed that trials in Block $1(M=977 \mathrm{~ms}, S E=18 \mathrm{~ms})$ were faster than trials in Block $2(M=1,110 \mathrm{~ms}, S E=20 \mathrm{~ms})$. A main effect of speedeffort, $\left.F(1,88)=40.30, p<.001, \eta_{\mathrm{p}}{ }^{2}=.314\right)$, showed that lowspeed-effort trials $(M=1,124 \mathrm{~ms}, S E=28 \mathrm{~ms})$ were slower than high-speed-effort trials $(M=963 \mathrm{~ms}, S E=12 \mathrm{~ms})$. All main effects were qualified by a PM Task Condition $\times$ Block interaction, $F(2,88)=71.95, p<.001, \eta_{\mathrm{p}}{ }^{2}=.621$, and by a Speed-Effort $\times$ Block interaction, $F(2,88)=19.47, p<.001$, $\eta_{\mathrm{p}}{ }^{2}=.181$. For the PM Task Condition $\times$ Block interaction, simple effects showed that RTs were faster in Block 1 relative to Block 2 for TAP $\left(M_{\mathrm{Blk} 1}=968 \mathrm{~ms}, S E_{\mathrm{Blk} 1}=30 \mathrm{~ms}, M_{\mathrm{Blk} 2}=\right.$ $\left.1,266 \mathrm{~ms}, S E_{\mathrm{Blk} 2}=33 \mathrm{~ms}\right)$ and TIP $\left(M_{\mathrm{Blk} 1}=995 \mathrm{~ms}, S E_{\mathrm{Blk} 1}=\right.$ $\left.30 \mathrm{~ms}, M_{\mathrm{Blk} 2}=1,159 \mathrm{~ms}, S E_{\mathrm{Blk} 2}=34 \mathrm{~ms}\right)$ conditions, and were slower in Block 1 relative to Block 2 for the control condition $\left(M_{\mathrm{Blk} 1}=967 \mathrm{~ms}, S E_{\mathrm{Blk} 1}=31 \mathrm{~ms}, M_{\mathrm{Blk} 2}=905 \mathrm{~ms}\right.$, $\left.S E_{\mathrm{Blk} 2}=34 \mathrm{~ms}\right), p \mathrm{~s}<.006$. For the Speed-Effort $\times$ Block interaction, simple effects showed that, overall, RTs were slower in Block 1 relative to Block 2 for low-speed-effort trials $\left(M_{\mathrm{Blk} 1}=1,075 \mathrm{~ms}, S E_{\mathrm{Blk} 1}=29 \mathrm{~ms}, M_{\mathrm{Blk} 2}=878 \mathrm{~ms}\right.$, $\left.S E_{\mathrm{Blk} 2}=10 \mathrm{~ms}\right)$ and high-speed-effort trials $\left(M_{\mathrm{Blk} 1}=\right.$ $1,173 \mathrm{~ms}, S E_{\mathrm{Blk} 1}=29 \mathrm{~ms}, M_{\mathrm{Blk} 2}=1,047 \mathrm{~ms}, S E_{\mathrm{Blk} 2}=$ $17 \mathrm{~ms}), p \mathrm{~s}<.001$. All other interactions were not significant, $p s>.675$.

For the living/nonliving ongoing task (in which the animal PM cue was considered TAP), a 3 (PM task condition: control vs. TAP vs. TIP) $\times 2$ (block: 1 vs. 2$) \times 2$ (speed-effort: low vs high) mixed-measures ANOVA with PM Task Condition as a between-subjects factor was performed on the RTs. A main 
Table 3 Baseline mean reaction times (RTs, in milliseconds), PM block mean RTs (in milliseconds), PM cue detection, ongoing task accuracy, and standard errors (in parentheses) by PM task condition and speed-effort for Experiment 2

\begin{tabular}{|c|c|c|c|c|}
\hline & Baseline Mean RT & PM Block Mean RT & PM Cue Detection & Ongoing Task Accuracy \\
\hline \multicolumn{5}{|c|}{ Consecutive-Letters Task } \\
\hline \multicolumn{5}{|l|}{ Control } \\
\hline Low & $1,068(44)$ & $968(49)$ & - & $.96(.01)$ \\
\hline Medium & $945(29)$ & $900(36)$ & - & $.96(.01)$ \\
\hline High & $866(26)$ & $843(37)$ & - & $.92(.01)$ \\
\hline \multicolumn{5}{|c|}{ TAP (Orthographic) } \\
\hline Low & $1,067(44)$ & $1,324(48)$ & $.70(.06)$ & $.97(.01)$ \\
\hline Medium & $956(29)$ & $1,246(35)$ & - & $.96(.01)$ \\
\hline High & $869(25)$ & $1,208(36)$ & $.60(.06)$ & $.94(.01)$ \\
\hline \multicolumn{5}{|c|}{ TIP (Semantic) } \\
\hline Low & $1,090(44)$ & $1,228(49)$ & $.65(.06)$ & $.94(.01)$ \\
\hline Medium & 989 (29) & $1,107(36)$ & - & $.97(.01)$ \\
\hline High & $900(26)$ & $1,089(37)$ & $.70(.06)$ & $.96(.01)$ \\
\hline \multicolumn{5}{|c|}{ Living/Nonliving Task } \\
\hline \multicolumn{5}{|l|}{ Control } \\
\hline Low & $1,170(44)$ & $1,072(48)$ & - & $.93(.01)$ \\
\hline Medium & $1,065(29)$ & $1,073(35)$ & - & $.93(.01)$ \\
\hline High & $1,014(25)$ & $1,018(36)$ & - & $.94(.01)$ \\
\hline \multicolumn{5}{|c|}{ TAP (Semantic) } \\
\hline Low & $1,110(44)$ & $1,234(49)$ & $.75(.06)$ & $.91(.01)$ \\
\hline Medium & $1,022(29)$ & $1,153(36)$ & - & $.94(.01)$ \\
\hline High & $956(26)$ & $1,139(37)$ & $.78(.06)$ & $.93(.01)$ \\
\hline \multicolumn{5}{|c|}{ TIP (Orthographic) } \\
\hline Low & $1,088(44)$ & $1,376(49)$ & $.63(.05)$ & $.92(.01)$ \\
\hline Medium & $1,012(29)$ & $1,251(36)$ & - & $.95(.01)$ \\
\hline High & 977 (26) & $1,278(37)$ & $.42(.06)$ & $.92(.01)$ \\
\hline
\end{tabular}

effect of block, $\left.F(1,88)=59.144, p<.001, \eta_{\mathrm{p}}{ }^{2}=.402\right)$, showed that trials in Block $1(M=1,053 \mathrm{~ms}, S E=17 \mathrm{~ms})$ were faster than trials in Block $2(M=1,186 \mathrm{~ms}, S E=25 \mathrm{~ms})$. A main effect of effort, $F(1,88)=69.18, p<.001, \eta_{\mathrm{p}}{ }^{2}=.44$, showed that low-speed-effort trials $(M=1,175 \mathrm{~ms}, S E=$ $22 \mathrm{~ms})$ were slower than high-speed-effort trials $(M=$ $1,064 \mathrm{~ms}, S E=19 \mathrm{~ms}$ ). All main effects were qualified by a PM Task Condition $\times$ Block interaction, $F(2,88)=$ 32.77, $\left.p<.001, \eta_{\mathrm{p}}{ }^{2}=.427\right)$, and by a Speed-Effort $\times$ Block interaction, $F(2,88)=8.47, p<.001, \eta_{\mathrm{p}}{ }^{2}=.088$. For the PM Task Condition $\times$ Block interaction, simple effects showed that RTs were faster in Block 1 relative to Block 2 for $\operatorname{TAP}\left(M_{\mathrm{Blk} 1}=1,033 \mathrm{~ms}, S E_{\mathrm{Blk} 1}=30 \mathrm{~ms}\right.$, $\left.M_{\mathrm{Blk} 2}=1,186 \mathrm{~ms}, S E_{\mathrm{Blk} 2}=43 \mathrm{~ms}\right)$ and $\mathrm{TIP}\left(M_{\mathrm{Blk} 1}=\right.$ $1,033 \mathrm{~ms}, S E_{\mathrm{Blk} 1}=30 \mathrm{~ms}, M_{\mathrm{Blk} 2}=1,327 \mathrm{~ms}, S E_{\mathrm{Blk} 2}=$ $43 \mathrm{~ms}$ ) conditions, $p \mathrm{~s}<.001$. The RTs for trials in Block 1 and Block 2 in the control condition did not differ significantly from each other, $p=.117$. For the SpeedEffort $\times$ Block interaction, simple effects showed that, overall, RTs were slower in Block 1 than in Block 2 for both low-speed-effort $\left(M_{\mathrm{Blk} 1}=1,122 \mathrm{~ms}, S E_{\mathrm{Blk} 1}=21 \mathrm{~ms}\right.$, $\left.M_{\mathrm{Blk} 2}=983 \mathrm{~ms}, S E_{\mathrm{Blk} 2}=18 \mathrm{~ms}\right)$ and high-speed-effort
$\left(M_{\mathrm{Blk} 1}=1,227 \mathrm{~ms}, S E_{\mathrm{Blk} 1}=27 \mathrm{~ms}, M_{\mathrm{Blk} 2}=1,145 \mathrm{~ms}\right.$, $\left.S E_{\mathrm{Blk} 2}=25 \mathrm{~ms}\right)$ trials, $p \mathrm{~s}<.001$. All other main effects and interactions were not significant, $p \mathrm{~s}>.07$.

$R T$ distribution analyses The procedure for conducting the distributional analysis was identical to Experiment 1. Mean parameter estimates are provided in Table 4 . The $\mu$ and $\tau$ parameter estimates were separately analyzed in a 3 (PM task condition: control vs. TAP vs. TIP) $\times 2$ (block: 1 vs. 2) $\times 2$ (speed-effort: low vs. high) mixedmeasures ANOVA with PM Task Condition as a betweensubjects factor. We omit report of analyses of the $\sigma$ parameters for parsimony. Furthermore, the $\sigma$ parameters are of less theoretical interest than the other parameters and no effects were found in the analyses of $\sigma$ in Experiment 1. The model fits converged for all data sets in Experiment 2. Thus, we had a complete set of parameter estimates for 182 participants. As was done for reporting the results for ongoing task speed, we conducted separate analyses for each ongoing task. Only significant effects are reported and further discussion of these results appears in the General Discussion. 
Table 4 Mean $\mu, \sigma$, and $\tau$ parameter estimates (in milliseconds), with standard errors (in parentheses), by PM task condition, speedeffort, and block for Experiment 2

\begin{tabular}{|c|c|c|c|c|c|c|}
\hline & \multicolumn{6}{|c|}{ Mean Parameter Estimates } \\
\hline & \multicolumn{2}{|l|}{$\mu$} & \multicolumn{2}{|l|}{$\sigma$} & \multicolumn{2}{|l|}{$\tau$} \\
\hline & Block 1 & Block 2 & Block 1 & Block 2 & Block 1 & Block 2 \\
\hline \multicolumn{7}{|l|}{ Consecutive Letters } \\
\hline Baseline/Low & $923(26)$ & $862(30)$ & $104(14)$ & $69(16)$ & $203(19)$ & $160(23)$ \\
\hline Baseline/Medium & $853(16)$ & $820(20)$ & $85(7)$ & $63(11)$ & $154(14)$ & $170(29)$ \\
\hline Baseline/High & $825(12)$ & $792(18)$ & $60(7)$ & $75(15)$ & $116(14)$ & $138(22)$ \\
\hline TAP/Low & $903(27)$ & $984(30)$ & $106(14)$ & $121(16)$ & $193(19)$ & $293(23)$ \\
\hline TAP/Medium & $839(16)$ & $936(20)$ & $69(7)$ & $103(11)$ & $148(14)$ & $263(19)$ \\
\hline TAP/High & $799(12)$ & $934(20)$ & $52(7)$ & $135(15)$ & $131(14)$ & $247(22)$ \\
\hline TIP/Low & $898(27)$ & $1,026(30)$ & $81(14)$ & $133(16)$ & $203(19)$ & $287(23)$ \\
\hline TIP/Medium & $867(16)$ & $928(20)$ & $68(7)$ & $86(11)$ & $131(14)$ & $257(19)$ \\
\hline TIP/High & $821(22)$ & 947 (19) & $59(19)$ & $118(15)$ & $117(14)$ & $242(22)$ \\
\hline \multicolumn{7}{|l|}{ Living/Nonliving } \\
\hline Baseline/Low & $927(30)$ & $884(32)$ & $119(21)$ & $78(15)$ & $248(27)$ & $189(34)$ \\
\hline Baseline/Medium & 895 (17) & $868(27)$ & $96(10)$ & $77(16)$ & $175(22)$ & 207 (27) \\
\hline Baseline/High & $878(17)$ & $843(27)$ & $62(11)$ & $97(21)$ & $138(22)$ & $172(35)$ \\
\hline TAP/Low & $875(30)$ & $952(32)$ & $100(22)$ & $88(20)$ & $246(27)$ & $261(34)$ \\
\hline TAP/Medium & $846(17)$ & $922(22)$ & $67(10)$ & $82(16)$ & $171(22)$ & $226(27)$ \\
\hline TAP/High & $829(17)$ & 937 (27) & $53(11)$ & $100(22)$ & $157(23)$ & $205(35)$ \\
\hline TIP/Low & $881(30)$ & $1,063(32)$ & $81(22)$ & $153(20)$ & $217(27)$ & $329(34)$ \\
\hline TIP/Medium & $864(17)$ & $928(27)$ & $62(10)$ & $88(16)$ & $152(22)$ & $330(27)$ \\
\hline TIP/High & $839(17)$ & $996(27)$ & $62(10)$ & $155(22)$ & $137(23)$ & $284(35)$ \\
\hline
\end{tabular}

Estimates for Block 1 and Block 2 are presented in the left and right columns for each parameter, respectively

We begin with the analyses for the consecutive-letters ongoing task. For the parameter estimates of $\mu$, a main effect of PM task type, $F(2,179)=5.53, p=.005, \eta_{\mathrm{p}}{ }^{2}=.058$, showed that trial RTs in the control block $(M=851 \mathrm{~ms}$, $S E=16 \mathrm{~ms})$ were faster than in both the TAP $(M=905 \mathrm{~ms}$, $S E=16 \mathrm{~ms}, p=.05)$ and TIP $(M=923 \mathrm{~ms}, S E=16 \mathrm{~ms}$, $p=.005)$ blocks. A main effect of block, $F(1,179)=48.49$, $p<.001, \eta_{\mathrm{p}}{ }^{2}=.217$, showed that trials in Block $1(M=862 \mathrm{~ms}$, $S E=9 \mathrm{~ms})$ were faster than trials in Block $2(M=924 \mathrm{~ms}$, $S E=11 \mathrm{~ms})$. A main effect of speed-effort, $F(1,179)=28.57$, $p<.001, \eta_{\mathrm{p}}{ }^{2}=.138$, showed that low-speed-effort trials $(M=933 \mathrm{~ms}, S E=15 \mathrm{~ms})$ were slower than high-speed-effort trials $(M=853 \mathrm{~ms}, S E=8 \mathrm{~ms})$. All main effects were qualified by a PM Task Condition $\times$ Block interaction, $F(2,179)=38.63$, $p<.001, \eta_{\mathrm{p}}{ }^{2}=.301$, and by a marginal Speed-Effort $\times$ Block interaction, $F(2,179)=3.59, p=.06, \eta_{\mathrm{p}}{ }^{2}=.02$. For the PM Task Condition $\times$ Block interaction, simple effects showed that RTs were faster in Block 1 than in Block 2 for both the TAP $\left(M_{\mathrm{Blk} 1}=851 \mathrm{~ms}, S E_{\mathrm{Blk} 1}=16 \mathrm{~ms}, M_{\mathrm{Blk} 2}=959 \mathrm{~ms}, S E_{\mathrm{Blk} 2}=\right.$ $20 \mathrm{~ms})$ and TIP $\left(M_{\mathrm{Blk} 1}=860 \mathrm{~ms}, S E_{\mathrm{Blk} 1}=16 \mathrm{~ms}, M_{\mathrm{Blk} 2}=\right.$ $\left.986 \mathrm{~ms}, S E_{\mathrm{Blk} 2}=20 \mathrm{~ms}\right)$ conditions, and were slower in Block 1 than in Block 2 for the control condition $\left(M_{\mathrm{Blk} 1}=874 \mathrm{~ms}\right.$,
$\left.S E_{\mathrm{Blk} 1}=16 \mathrm{~ms}, M_{\mathrm{Blk} 2}=827 \mathrm{~ms}, S E_{\mathrm{Blk} 2}=20 \mathrm{~ms}\right), p \mathrm{~s}<.002$. For the marginal Speed-Effort $\times$ Block interaction, simple effects showed that, overall, RTs were slower in Block 1 than in Block 2 for both low-speed-effort trials $\left(M_{\mathrm{Blk} 1}=\right.$ $\left.908 \mathrm{~ms}, S E_{\mathrm{Blk} 1}=15 \mathrm{~ms}, M_{\mathrm{Blk} 2}=815 \mathrm{~ms}, S E_{\mathrm{Blk} 2}=7 \mathrm{~ms}\right)$ and high-speed-effort trials $\left(M_{\mathrm{Blk} 1}=957 \mathrm{~ms}, S E_{\mathrm{Blk} 1}=\right.$ $\left.17 \mathrm{~ms}, M_{\mathrm{Blk} 2}=891 \mathrm{~ms}, S E_{\mathrm{Blk} 2}=11 \mathrm{~ms}\right), p \mathrm{~s}<.005$. All other interactions were not significant, $p \mathrm{~s}>.251$

For the parameter estimates of $\tau$, a main effect of PM task type, $F(2,179)=6.31, p=.002, \eta_{\mathrm{p}}{ }^{2}=.066$, showed that trial RTs in the control block ( $M=154 \mathrm{~ms}, S E=14 \mathrm{~ms})$ had smaller $\tau \mathrm{s}$ than those in either the TAP $(M=216 \mathrm{~ms}, S E=14 \mathrm{~ms}$, $p=.005)$ or the TIP $(M=212 \mathrm{~ms}, S E=14 \mathrm{~ms}, p=.01)$ block. A main effect of block, $F(1,179)=34.38, p<.001, \eta_{\mathrm{p}}{ }^{2}=.161$, showed that trials in Block $1(M=160 \mathrm{~ms}, S E=8 \mathrm{~ms})$ had smaller $\tau$ s than did trials in Block $2(M=228 \mathrm{~ms}, S E=11 \mathrm{~ms})$. A main effect of effort, $F(1,179)=43.99, p<.001, \eta_{\mathrm{p}}{ }^{2}=.002$, showed that low-speed-effort trials $(M=223 \mathrm{~ms}, S E=10 \mathrm{~ms})$ had larger $\tau$ s than did high-speed-effort trials $(M=165 \mathrm{~ms}$, $S E=9 \mathrm{~ms}$ ). All main effects were qualified by a PM Task Condition $\times$ Block interaction, $F(2,179)=11.52, p<.001$, $\eta_{\mathrm{p}}{ }^{2}=.114$, and by a Speed-Effort $\times$ Block interaction, 
$F(2,179)=5.89, p<.001, \eta_{\mathrm{p}}{ }^{2}=.032$. For the PM Task Condition $\times$ Block interaction, simple effects showed that RTs had smaller $\tau$ s in Block 1 than in Block 2 for the TAP $\left(M_{\mathrm{Blk} 1}=161 \mathrm{~ms}, S E_{\mathrm{Blk} 1}=14 \mathrm{~ms}, M_{\mathrm{Blk} 2}=149 \mathrm{~ms}, S E_{\mathrm{Blk} 2}\right.$ $=20 \mathrm{~ms}, p<.001)$ and TIP $\left(\mathrm{M}_{\mathrm{Blk} 1}=160 \mathrm{~ms}, S E_{\mathrm{Blk} 1}=14 \mathrm{~ms}\right.$, $\left.M_{\mathrm{Blk} 2}=264 \mathrm{~ms}, S E_{\mathrm{Blk} 2}=20 \mathrm{~ms}, p<.001\right)$ conditions, but no differences in $\tau$ s by block for the control condition, $p=.599$. For the Speed-Effort $\times$ Block interaction, simple effects showed that, overall, $\tau$ s were larger in Block 1 than in Block 2 for low-speed-effort trials ( $M_{\mathrm{Blk} 1}=200 \mathrm{~ms}, S E_{\mathrm{Blk} 1}=11 \mathrm{~ms}$, $\left.M_{\mathrm{Blk} 2}=121 \mathrm{~ms}, S E_{\mathrm{Blk} 2}=8 \mathrm{~ms}\right)$ and for high-speed-effort trials $\left(M_{\mathrm{Blk} 1}=247 \mathrm{~ms}, S E_{\mathrm{Blk} 1}=13 \mathrm{~ms}, M_{\mathrm{Blk} 2}=209 \mathrm{~ms}\right.$, $\left.S E_{\mathrm{Blk} 2}=13 \mathrm{~ms}\right), p \mathrm{~s}<.005$. All other interactions were not significant, $p \mathrm{~s}>.478$.

We now turn to the analyses for the living/nonliving ongoing task. For the parameter estimates of $\mu$, a marginal main effect of PM task type, $F(2,88)=2.55, p=.084, \eta_{\mathrm{p}}{ }^{2}=.055$, showed that trial RTs in the control block ( $M=883 \mathrm{~ms}, S E=20 \mathrm{~ms})$ were faster than those in the TAP ( $M=898 \mathrm{~ms}, S E=20 \mathrm{~ms})$ and TIP ( $M=945 \mathrm{~ms}, S E=20 \mathrm{~ms}$ ) blocks. A main effect of block, $F(1,88)=33.65, p<.001, \eta_{\mathrm{p}}{ }^{2}=.277$, showed that trials in Block $1(M=871 \mathrm{~ms}, S E=12 \mathrm{~ms})$ were faster than trials in Block $2(M=946 \mathrm{~ms}, S E=15 \mathrm{~ms})$. A main effect of speedeffort, $F(1,88)=10.30, p=.002, \eta_{\mathrm{p}}{ }^{2}=.105$, showed that lowspeed-effort trials $(M=930 \mathrm{~ms}, S E=16 \mathrm{~ms})$ were slower than high-speed-effort trials $(M=887 \mathrm{~ms}, S E=11 \mathrm{~ms})$. The main effects were qualified by a PM Task Condition $\times$ Block interaction, $F(2,88)=22.91, p<.001, \eta_{\mathrm{p}}{ }^{2}=.342$. For this interaction, simple effects showed that RTs were faster in Block 1 than in Block 2 for TAP $\left(M_{\mathrm{Blk} 1}=852 \mathrm{~ms}, S E_{\mathrm{Blk} 1}=20 \mathrm{~ms}, M_{\mathrm{Blk} 2}\right.$ $\left.=945 \mathrm{~ms}, S E_{\mathrm{Blk} 2}=26 \mathrm{~ms}\right)$ and TIP $\left(M_{\mathrm{Blk} 1}=860 \mathrm{~ms}, S E_{\mathrm{Blk} 1}\right.$ $=20 \mathrm{~ms}, M_{\mathrm{Blk} 2}=1,029 \mathrm{~ms}, S E_{\mathrm{Blk} 2}=25 \mathrm{~ms}$ ) conditions (both $p \mathrm{~s},<.001)$, and were marginally slower in Block 1 than in Block 2 for the control condition $\left(M_{\mathrm{Blk} 1}=903 \mathrm{~ms}, S E_{\mathrm{Blk} 1}\right.$ $\left.=20 \mathrm{~ms}, M_{\mathrm{Blk} 2}=863 \mathrm{~ms}, S E_{\mathrm{Blk} 2}=25 \mathrm{~ms}\right), p=.076$.

For the parameter estimates of $\tau$, a main effect of block, $F(1,88)=7.96, p=.006, \eta_{\mathrm{p}}{ }^{2}=.083$, showed that trials in Block $1(M=190 \mathrm{~ms}, S E=12 \mathrm{~ms})$ had smaller $\tau \mathrm{s}$ than trials in Block $2(M=240 \mathrm{~ms}, S E=18 \mathrm{~ms})$. A main effect of speedeffort, $F(1,88)=33.14, p<.001, \eta_{\mathrm{p}}{ }^{2}=.274$, showed that lowspeed-effort trials $(M=248 \mathrm{~ms}, S E=14 \mathrm{~ms})$ had larger $\tau \mathrm{s}$ than high-speed-effort trials $(M=182 \mathrm{~ms}, S E=14 \mathrm{~ms})$. All main effects were qualified by a PM Task Condition $\times$ Block interaction, $F(2,88)=5.72, p=.005, \eta_{\mathrm{p}}{ }^{2}=.115$, and by a Speed-Effort $\times$ Block interaction, $F(2,88)=4.45, p=.038$, $\eta_{\mathrm{p}}{ }^{2}=.048$. For the PM Task Condition $\times$ Block interaction, simple effects showed that RTs had smaller $\tau$ s in Block 1 than in Block 2 for the TIP condition $\left(M_{\mathrm{Blk} 1}=177 \mathrm{~ms}, S E_{\mathrm{Blk} 1}\right.$ $\left.=21 \mathrm{~ms}, M_{\mathrm{Blk} 2}=307 \mathrm{~ms}, S E_{\mathrm{Blk} 2}=31 \mathrm{~ms}, p<.001\right)$, but no differences in $\tau$ s were apparent by blocks for the control and TAP conditions, $p>.301$. For the Effort $\times$ Block interaction, simple effects showed that, overall, $\tau \mathrm{s}$ were larger in Block 1 than in Block 2 for low-speed-effort trials $\left(M_{\mathrm{Blk} 1}=237 \mathrm{~ms}, S E_{\mathrm{Blk} 1}=16 \mathrm{~ms}, M_{\mathrm{Blk} 2}=144 \mathrm{~ms}\right.$, $\left.S E_{\mathrm{Blk} 2}=13 \mathrm{~ms}, p<.001\right)$, and smaller in Block 1 than in Block 2 for high-speed-effort trials $\left(M_{\mathrm{Blk} 1}=260 \mathrm{~ms}, S E_{\mathrm{Blk} 1}\right.$ $=20 \mathrm{~ms}, M_{\mathrm{Blk} 2}=220 \mathrm{~ms}, S E_{\mathrm{Blk} 2}=20 \mathrm{~ms}, p=.036$ ). All other interactions were not significant, $p \mathrm{~s}>.193$.

\section{General discussion}

The present study was designed to further investigate Marsh et al.'s (2005) inquiry into the role of attentional resources for PM performance in TAP conditions. Furthermore, considering the discrepancies between Marsh et al.'s and Meiser and Schult's (2008) results in addition to current views (e.g., the PAM view; MP view) on the role of attentional resources for PM performance, further research was warranted. Concurrent to the previously discussed purposes, the present study also extends current knowledge of the interactions between match in processing and attention allocation, particularly, when controlled processes are required.

In Experiment 1, the results suggest that participants utilized less controlled processes when there was a match in processing (TAP) and when there were sufficient amounts of attentional resources available for the PM task (low speed-effort). However, when there was a match in processing but fewer attentional resources were available for the PM task (high speed-effort) and in all mismatched processing conditions, participants relied on controlled processes such as strategic monitoring to a greater degree. Thus, there appears to be an interaction between processing match and attention allocation regarding processes utilized in PM tasks. However, this interaction was only apparent in the standard mean RT analysis and the $\mu$ parameter estimates analysis, not in the $\tau$ estimates analysis. The results from the RT distribution analyses do suggest, however, that the $\tau$ parameter may be diagnostic of the frequency of trials in which monitoring played a role, as evidenced by the larger slow tails in TIP conditions. Finally, consistent with predictions regarding processing overlap degree, PM performance was higher in the TAP than in the TIP conditions, a finding consistent with past studies that examined the TAP view (Maylor, 1998). In Experiment 2, PM accuracy results further supported these findings, showing a TAP advantage for both semantic and orthographic PM tasks, despite higher PM performance overall on the semantic PM tasks. For both ongoing task conditions (consecutive letter and living/nonliving), task interference was observed for TAP and TIP conditions, in which RTs were slower in Block 1 than in Block 2, whereas, for the control condition, no interference was observed. However, results from the $\tau$ parameter estimates of the ex-Gaussian distributional analysis suggest that controlled monitoring is a function not just of TAP/TIP, but also of ongoing task type. For the 
consecutive-letters ongoing task, observably more monitoring occurred in Block 2 than in Block 1 for both the TAP and TIP PM conditions. However, for the living/ nonliving ongoing task, evidence for monitoring was only shown in the TIP PM condition. We will expound upon this particular effect below.

Interactions between match in processing and attention allocation

One of the more important findings from this study is the observation that the match in processing and the amount of attention allocation interact. Task interference results showed that the relative amount of controlled processes participants utilized during PM tasks was determined by the varied conditions of match in processing and the amount of attention allocation. Specifically, during TIP/low, TIP/high, and TAP/high conditions in Experiment 1, participants relied on relatively more controlled processes such as strategic monitoring than did participants in TAP/low conditions. These results suggest that investigating the independent effects of processing conditions may overlook important conditional effects such as attention allocation.

Interactions between match in processing and ongoing task

Experiment 2 showed that the TAP advantage in PM accuracy did not depend on the type of PM task completed. However, the ongoing task speed analyses suggested that the nature of the ongoing/PM task overlap affects the level of controlled processes participants engage in. The $\tau$ analyses indicated different PM Task Condition $\times$ Block interactions. For the living/nonliving task (in which the TAP condition involved a semantic match in processing), results were similar to Experiment 1 with higher $\tau$ estimates for Block 2 than in Block 1 only for the TIP condition. For the consecutive-letters task (in which the TAP condition involved an orthographic match in processing), higher $\tau$ estimates were found for Block 2 than Block 1 for both TAP and TIP conditions. No block difference was seen in the control conditions for either ongoing task. If we take the $\tau$ parameter to reflect more "analytic" or controlled processing (see Balota \& Spieler, 1999), a tentative conclusion for the increase in $\tau$ during these conditions might be that more controlled processing strategies were needed for successful performance in the orthographic TAP condition, in which PM accuracy was also lower than for the semantic PM tasks. In other words, more controlled processing is needed when both the ongoing and PM tasks are more difficult, even if a match in processing is present. Further support for this comes from the observed effects of ongoing task type on PM performance in Experiment 2. PM accuracy varied as a function of ongoing task type. PM performance for orthographic PM cues depended on the type of ongoing task: Namely, improved performance was observed during the consecutive-letters ongoing task. PM performance for semantic PM cues did not depend on the type of ongoing task. These results provide further evidence that the properties of the PM/ongoing task overlap play a crucial part in both the success of PM cue detection and the type of processing that is utilized.

\section{PM performance modulated by TAP/TIP properties}

One of the primary goals of the present study was to further investigate the conflicting results in the PM literature regarding TAP/TIP effects. In two experiments, and controlling for attention allocation (i.e., speed-effort), we found evidence supporting the notion that successful PM performance depends on a match in processing. Thus, our results support the findings presented by Meiser and Schult (2008). Furthermore, these results were found after controlling for not only the effects of effort but also type of effort (i.e., speed-effort). This latter notion should help assist future PM researchers intending to implement an experimental manipulation of effort.

Moreover, differential TAP/TIP effects were also observed as a function of ongoing task type. As we discussed above, the nature (and difficulty) of the ongoing and PM tasks interact with the overall TAP/TIP effect and subsequently affect the success of PM performance. Interestingly, as inferred from the interaction between PM task type and ongoing task type in the $\tau$ parameter-which implicates variable controlled monitoring - semantic lexical decisions appear to be easier than orthographic lexical decisions.

It is important to note that the experimental design of the present study did not afford an optimal setting for investigating automatic processing. On the contrary, the design of the present study, and the utilization of the ex-Gaussian distribution analysis, provided more information regarding the potential (and amount) of controlled processes that participants used for successful PM performance. Indeed, particular theories of PM purport different processing strategies (e.g., multiprocess theory's spontaneous retrieval) that could loosely be generalized by automatic versus controlled processes depending on other task properties and constraints (e.g., focal vs. nonfocal). Meiser and Schult (2008) provide an argument for automatic processing in TAP conditions. We also suggested that the notion of match in processing of ongoing and PM tasks maintained by previous research (Marsh et al., 2005; Maylor, 1996, 1998; Meiser \& Schult, 2008) could be viewed as a weak form of focal processing (see Einstein \& McDaniel, 2005). Although our study was not designed to examine conditions under which automatic processing occurs in PM tasks, a tentative conclusion is that more automatic processing occurs in the conditions for which we found less task inference (i.e., TAP conditions 
with a semantic match in processing). Future research should attend to the open question of the trade-off of automatic/controlled processing while considering the diverse task constraints presented in the present study. Likewise, we believe that the implementation of the ex-Gaussian distribution analysis is a potentially useful tool for investigating and expanding current theoretical issues in PM research (see Brewer, 2011; McBride \& Abney, 2012).

\section{Conclusions}

Consistent with predictions of TAP in PM tasks, a match in processing for ongoing and PM tasks enhanced PM cue detection relative to a mismatch in processing. Manipulations of attention allocation did not influence PM cue detection. However, ongoing task speed varied as a function of the amount of attention allocation and degree of processing overlap in PM task sets. Specifically, we observed more reliance on controlled processes (such as strategic monitoring) for PM task sets with a match in processing and high attention allocation (Exp. 1), for PM task sets with a match in processing and an orthographic ongoing task (Exp. 2), and for PM task sets with a mismatch in processing (Exps. 1 and 2). Distributional analyses of ongoing task performance provided additional support for the overall finding that the reliance on controlled processes, to some degree, is a function of the processing overlap between the ongoing and PM tasks. We conclude that the reliance on controlled processes for successful PM performance is a function of the processing overlap, PM cue properties, and the amount of attentional resources.

Author note D.A. is now at the University of California, Merced. We thank Courtney Schreiner for assistance with data collection and coding. We also thank Gene Brewer and Hunter Ball for helpful comments on a previous version of this manuscript.

\section{References}

Balota, D. A., \& Spieler, D. H. (1999). Word frequency, repetition, and lexicality effects in word recognition tasks: Beyond measures of central tendency. Journal of Experimental Psychology. General, 128, 32-55. doi:10.1037/0096-3445.128.1.32

Balota, D. A., \& Yap, M. J. (2011). Moving beyond the mean in studies of mental chronometry: The power of response time distributional analyses. Current Directions in Psychological Science, 20, 160-166.

Brewer, G. A. (2011). Analyzing response time distributions: Methodological and theoretical suggestions for prospective memory researchers. Journal of Psychology, 219, 117-124.

Chism, S. J. (1992). From A to zotamorf: The dictionary of palindromes. Morristown, NJ: Word Ways Press.

Cousineau, D., Brown, S. D., \& Heathcote, A. (2004). Fitting distributions using maximum likelihood: Methods and packages. Behavior Research Methods, Instruments, \& Computers, 36, 742-756. doi:10.3758/BF03206555
Einstein, G. O., \& McDaniel, M. A. (1990). Normal aging and prospective memory. Journal of Experimental Psychology: Learning, Memory, and Cognition, 16, 717-726. doi:10.1037/02787393.16.4.717

Einstein, G. O., \& McDaniel, M. A. (2005). Prospective memory: Multiple retrieval processes. Current Directions in Psychological Science, 14, 286-290. doi:10.1111/j.0963-7214.2005.00382.x

Einstein, G. O., McDaniel, M. A., Thomas, R., Mayfield, S., Shank, H., Morrisette, N., \& Breneiser, J. (2005). Multiple processes in prospective memory retrieval: Factors determining monitoring versus spontaneous retrieval. Journal of Experimental Psychology. General, 134, 327-342. doi:10.1037/0096-3445.134.3.327

Ellis, J. A. (1996). Prospective memory or the realization of delayed intentions: A conceptual framework for research. In M. Brandimonte, G. O. Einstein, \& M. A. McDaniel (Eds.), Prospective memory: Theory and applications (pp. 1-22). Mahwah, NJ: Erlbaum.

Heathcote, A., Brown, S. D., \& Cousineau, D. (2004). QMPE: Estimating Lognormal, Wald, and Weibull RT distributions with a parameter dependent lower bound. Behavior Research Methods, Instruments, \& Computers, 36, 277-290. doi:10.3758/BF03195574

Heathcote, A., Popiel, S. J., \& Mewhort, D. J. (1991). Analysis of response time distributions: An example using the Stroop task. Psychological Bulletin, 109, 340-347. doi:10.1037/00332909.109.2.340

Hohle, R. H. (1965). Inferred components of reaction times as functions of foreperiod duration. Journal of Experimental Psychology, 69, 382-386. doi:10.1037/h0021740

Kučera, H., \& Francis, W. N. (1967). Computational analysis of present-day American English. Providence, RI: Brown University Press.

Luce, R. D. (1986). Response times: Their role in inferring mental organization. New York, NY: Oxford University Press.

Marsh, R. L., Hicks, J. L., \& Cook, G. I. (2005). On the relationship between effort toward an ongoing task and cue detection in eventbased prospective memory. Journal of Experimental Psychology: Learning, Memory, and Cognition, 31, 68-75. doi:10.1037/02787393.31.1.68

Marsh, R. L., Hicks, J. L., \& Hancock, T. W. (2000). On the interaction of ongoing cognitive activity and the nature of an event-based intention. Applied Cognitive Psychology, 14, S29-S41.

Matzke, D., \& Wagenmakers, E.-J. (2009). Psychological interpretation of the ex-Gaussian and shifted Wald parameters: a diffusion model analysis. Psychonomic Bulletin \& Review, 16, 798-817. doi:10.3758/PBR.16.5.798

Maylor, E. A. (1996). Age-related impairment in an event-based prospective-memory task. Psychology and Aging, 11, 74-78.

Maylor, E. A. (1998). Changes in event-based prospective memory across adulthood. Aging, Neuropsychology, and Cognition, 5, 107-128.

McBride, D. M., \& Abney, D. H. (2012). A comparison of transferappropriate processing and multiprocess frameworks for prospective memory performance. Experimental Psychology, 59, 190-198.

McDaniel, M. A., \& Einstein, G. O. (2000). Strategic and automatic processes in prospective memory retrieval: A multiprocess framework. Applied Cognitive Psychology, 14, S127-S144. doi:10.1002/acp.775

McGann, D., Ellis, J., \& Milne, A. (2002). Conceptual and perceptual processes in prospective remembering: Differential influence of attentional resources. Memory \& Cognition, 30, 1021-1032.

McGann, D., Ellis, J., \& Milne, A. (2003). Conceptual and perceptual processing in prospective remembering. European Journal of Cognitive Psychology, 15, 19-41.

McGill, W. J., \& Gibbon, J. (1965). The general-gamma distribution and reaction times. Journal of Mathematical Psychology, 2, 1-18. 
Meier, B., \& Graf, P. (2000). Transfer appropriate processing for prospective memory tests. Applied Cognitive Psychology, 14, S11-S27.

Meiser, T., \& Schult, J. C. (2008). On the automatic nature of the taskappropriate processing effect in event-based prospective memory. European Journal of Cognitive Psychology, 20, 290-311.

Morris, C. D., Bransford, J. D., \& Franks, J. J. (1977). Levels of processing versus transfer appropriate processing. Journal of Verbal Learning and Verbal Behavior, 16, 519-533. doi:10.1016/ S0022-5371(77)80016-9

Rastle, K., Harrington, J., \& Coltheart, M. (2002). 358,534 nonwords: The ARC Nonword Database. Quarterly Journal of Experimental Psychology, 55A, 1339-1362. doi:10.1080/02724980244000099

Roediger, H. L., III, Weldon, M. S., \& Challis, B. H. (1989). Explaining dissociations between implicit and explicit measures of retention: A processing account. In H. L. Roediger III \& F. I. M. Craik (Eds.), Varieties of memory and consciousness: Essays in honour of Endel Tulving (pp. 3-39). Hillsdale, NJ: Erlbaum.

Smith, R. E. (2003). The cost of remembering to remember in eventbased prospective memory: Investigating the capacity demands of delayed intention performance. Journal of Experimental Psychology: Learning, Memory, and Cognition, 29, 347-361.

Tse, C.-S., Balota, D. A., Yap, M. J., Duchek, J. M., \& McCabe, D. P. (2010). Effects of healthy aging in early stage dementia of the Alzheimer's type on components of response time distributions in three attention tasks. Neuropsychology, 24, 300-315.

West, R., \& Craik, F. I. M. (2001). Influences on the efficiency of prospective memory in younger and older adults. Psychology and Aging, 16, 682-696. 